\title{
Performance Tolerance of IMDD DFMA PONs to Channel Frequency Response Roll-off
}

Giddings, Roger; Duan, Xiao; Mansoor, Saad; Tang, Jianming

\section{IEEE Photonics Technology Letters}

\author{
DOI: \\ 10.1109/LPT.2017.2740560
}

Published: 01/10/2017

Peer reviewed version

Cyswllt i'r cyhoeddiad / Link to publication

Dyfyniad o'r fersiwn a gyhoeddwyd / Citation for published version (APA):

Giddings, R., Duan, X., Mansoor, S., \& Tang, J. (2017). Performance Tolerance of IMDD DFMA PONs to Channel Frequency Response Roll-off. IEEE Photonics Technology Letters, 29(19), 1655-1658. https://doi.org/10.1109/LPT.2017.2740560

\footnotetext{
Hawliau Cyffredinol / General rights

Copyright and moral rights for the publications made accessible in the public portal are retained by the authors and/or other copyright owners and it is a condition of accessing publications that users recognise and abide by the legal requirements associated with these rights.

- Users may download and print one copy of any publication from the public portal for the purpose of private study or research.

- You may not further distribute the material or use it for any profit-making activity or commercial gain

- You may freely distribute the URL identifying the publication in the public portal?
}

Take down policy

If you believe that this document breaches copyright please contact us providing details, and we will remove access to the work immediately and investigate your claim. 


\title{
Performance Tolerance of IMDD DFMA PONs to Channel Frequency Response Roll-off
}

\author{
X. Duan, R. P. Giddings, Member, IEEE, S. Mansoor and J. M. Tang, Member, IEEE
}

\begin{abstract}
The impacts of the channel frequency response rolloff effect on upstream optical OFDM transmission performance in digital filter multiple access (DFMA) passive optical networks (PONs) based on intensity modulation and direct detection (IMDD) are investigated, in terms of BER versus received optical power performance, ONU transmission capacity, ONU launch power variation (LPV) range and inter-ONU sample timing offset (STO). The effect of signal bandwidth is also explored by comparing tolerance to the channel roll-off effect for two different signal bandwidths. Over all of the aforementioned aspects, good agreements are obtained between numerical simulations and experimental measurements. The results provide important insights into channel roll-off-induced performance dependencies to facilitate cost-effective designs of both DFMA transceivers and IMDD DFMA PONs.
\end{abstract}

Index Terms - Digital signal processing, passive optical networks, digital orthogonal filters and digital filter multiple access.

\section{INTRODUCTION}

$I$ $\mathrm{t}$ is well known that traditional passive optical networks (PONs) are becoming ill-suited to efficiently accommodate the emerging highly dynamic data traffic patterns. Furthermore, future PONs are also envisaged to be equipped with softwaredefined networking (SDN) $[1,2]$ to provide reconfigurable and elastic on-demand interconnects down to the physical layer. To deliver such desired functionalities with sufficient transparency to key network design features, we have recently proposed a new multiple access technique termed digital filter multiple access (DFMA) [3], which uses digital signal processing (DSP)-based, SDN-controllable and transceiver-embedded digital orthogonal filters to enable dynamically reconfigurable and elastic physical interconnections between ONUs and the OLT to meet transient network traffic demands. The operating principle and major advantages of DFMA-PONs over traditional PONs are fully outlined in [4]. The following key features should be highlighted. Dynamically reconfigurable and elastic architectures at the subwavelength level support physical layer SDN network virtualization and abstraction. There is also transparency to underlying modulation formats, multiple access methods, signal bandwidths etc. Furthermore, cloud access

Manuscript received June 13, 2017; revised July 28, 2017; accepted August 04, 2017. Date of publication August ..., 2017; date of current version August ..., 2017.

X. Duan, R. P. Giddings (e-mail: r.p.giddings@bangor.ac.uk), and J. M. Tang are with the School of Electronic Engineering, Bangor University, Bangor LL57 1UT, UK.

S. Mansoor is with the School of Computer Science, Bangor University, Bangor LL57 1UT, UK

Copyright (C) 2017 IEEE networks (CANs) can be realised to concurrently support both fixed and mobile traffic, facilitated by DFMA-enabled subwavelength, flexible and reconfigurable optical add/drop multiplexers [5], allowing rapid all-optical switching/routing of DFMA-channels within the CAN. Network reconfiguration by a SDN controller is also simply achieved by reprogramming the DFMA filter coefficients via the use of a transceiver embedded DSP controller and a control protocol such as OpenFlow [2].

To validate the DFMA-PON's feasibility, multipoint-topoint optical OFDM (OOFDM) upstream transmissions of IMDD DFMA PONs [4] have recently been successfully experimentally demonstrated, where Hilbert pair-based digital orthogonal filters are assigned to two spectrally overlapped orthogonal channels occupying a common spectral region. As such, the channel frequency response characteristics influenced the orthogonality between the channel pair, leading to crosschannel interference (CCI) and subsequent system performance degradation [6]. To effectively mitigate the channel frequency response-induced CCI, a DSP-based CCI cancellation (CCIC) technique has been proposed and experimentally demonstrated in a point-to-point IMDD DFMA PON system [6]. Also a more comprehensive DSP-based DFMA channel interference cancellation (DCIC) technique has been numerically investigated in multipoint-to-point IMDD DFMA PONs [7].

As component, transceiver and system designs ultimately determine total system frequency response, it is highly beneficial to explore the IMDD DFMA PON performance robustness to various levels of channel frequency response rolloff (here on "channel roll-off”), as such studies can: i) offer insights into optimum PON operating conditions where CCIC becomes unnecessary, allowing further simplifications of both transceivers and PON architectures without considerably compromising the overall PON performance; and ii) indicate the feasibility of utilising low-cost and narrowband electrical and optical components. This is essential for achieving the optimum balance between PON installation and operation costs and the required PON performance. To explore the aforementioned performance robustness, this Letter details performance comparisons between experimental measurements and numerically simulated results, and analyses the impacts of channel roll-off on upstream IMDD DFMA PONs in terms of BER versus received optical power (ROP), maximum achievable ONU capacity, ONU launch power variation (LPV) range, and tolerance to inter-ONU sampling time offset (STO). Here we focus on upstream PON performance as this is subject to more variations in its operating conditions, resulting from the 
independently operating ONUs. Furthermore, a two ONU PON is considered sufficient for the analysis as roll-off induced CCI is only dependent on roll-off within orthogonal sub-bands [7].

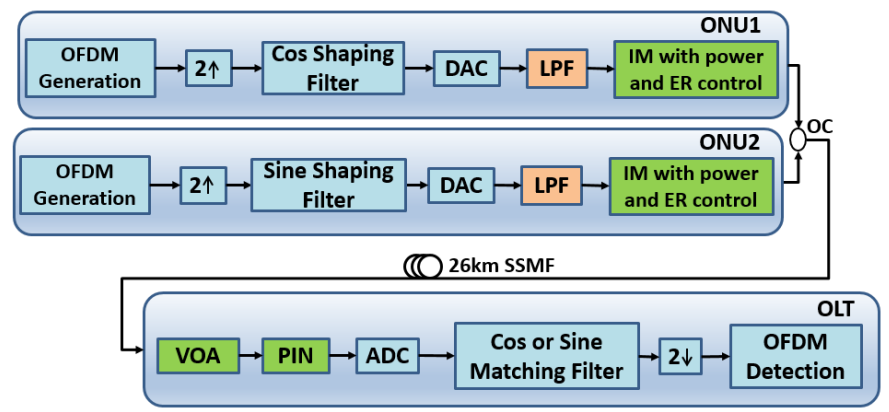

Fig. 1. DFMA PON system model adopted in the simulation (DAC: digital-to-analogue converter, ADC: analogue-to-digital converter, OC: optical coupler, VOA: variable optical attenuator)

\section{IMDD DFMA PONS}

Fig.1 illustrates the considered two ONU IMDD DFMA PON, which is identical to that employed in experimental measurements [4], except that in Fig.1 an electrical low pass filter (LPF) is introduced in each ONU to take into account the roll-off effect attributed by relevant analogue electrical and optical components. As shown in Fig.1, the upstream signal DSP and transmission processes can be described as follows: in each ONU transmitter, a digital OFDM signal is firstly generated and then $2 \times$ up-sampled by adding a zero-valued sample between two consecutive original samples. The upsampled digital signal is filtered by a 32-tap finite impulse response (FIR) digital shaping filter to generate an in-phase signal for ONU1 (cosine filter) or a quadrature-phase signal for ONU2 (sine filter). After passing through a 2GS/s, 8-bit DAC and the LPF, the electrical signal is utilized to drive an intensity modulator (IM) in the corresponding ONU. Each ONU's optical signal power is fixed at $4 \mathrm{dBm}$. After passively combining these two upstream DFMA signals in a $3 \mathrm{~dB}$ optical coupler, the combined optical signals propagate along a $26 \mathrm{~km}$ SSMF to the OLT. In the OLT, the ROP of the optical signal is adjusted with a variable optical attenuator. The received optical signal is detected with a $12.5 \mathrm{GHz}$ PIN with a receiver sensitivity of $-19 \mathrm{dBm}$. The detected electrical analogue signal is converted into the digital domain using a 2GS/s, 8-bit ADC. Finally, the major OLT receiver DSP procedures include: filtering by a digital matching filter selected according to the

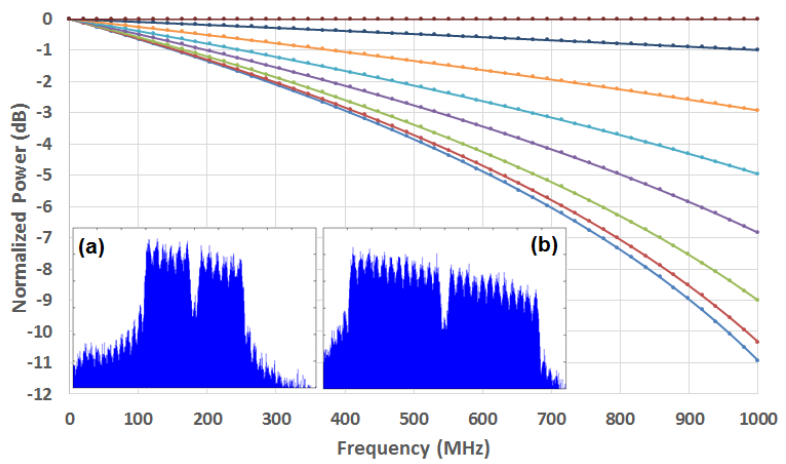

Fig. 2. Different frequency response roll-offs introduced by the LPFs. Inset: DFMA electrical signal spectra at $R=10 \mathrm{~dB}$ (a) $\alpha=0.43$, (b) $\alpha=0.81$
TABLE I

SYSTEM PARAMETERS

\begin{tabular}{|c|c|c|}
\hline Parameter & Value & Unit \\
\hline Total number of IFFT/FFT points & 32 & I \\
\hline Data-carrying subcarriers (high frequencies) & 6 or 12 & l \\
\hline Subcarrier modulation format & 16-QAM & I \\
\hline Subcarrier frequency spacing & 31.25 & $\mathrm{MHz}$ \\
\hline Up/down-sampling factor & 2 & I \\
\hline Digital filter length & 16 & I \\
\hline OFDM symbol rate & 25 & $\mathrm{MHz}$ \\
\hline OFDM symbol samples: Data ${ }^{\alpha} /$ Cyclic prefix $^{\alpha}$ & $32 / 8$ & samples \\
\hline OFDM signal clipping level & 13 & $\mathrm{~dB}$ \\
\hline DAC/ADC sample rate & 2 & GS/s \\
\hline DAC/ADC resolution & 8 & bit \\
\hline Excess of bandwidth of digital filters & 0 & / \\
\hline Orthogonal sub-band bandwidth & 1 & $\mathrm{GHz}$ \\
\hline Total raw bit rate (6 - 12 subcarriers/channel) & $1.5-3.0$ & $\mathrm{~Gb} / \mathrm{s}$ \\
\hline Extinction ratio of IMs & 0.49 & $\mathrm{~dB}$ \\
\hline PIN quantum efficiency & 0.8 & l \\
\hline PIN power sensitivity ${ }^{\beta}$ & -19 & $\mathrm{dBm}$ \\
\hline PIN detection bandwidth & 12.5 & $\mathrm{GHz}$ \\
\hline Fiber dispersion parameter & 17 & $\mathrm{ps} / \mathrm{nm} / \mathrm{km}$ \\
\hline Fiber dispersion slope & 0.07 & $\mathrm{ps} / \mathrm{nm}^{2} / \mathrm{km}$ \\
\hline Fiber loss & 0.2 & $\mathrm{~dB} / \mathrm{km}$ \\
\hline Fiber Kerr coefficient & $2.35 \times 10^{-20}$ & $\mathrm{~m}^{2} / \mathrm{W}$ \\
\hline
\end{tabular}

$\alpha$ Before up-sampling / after down-sampling

$\beta$ Corresponding to $10 \mathrm{~Gb} / \mathrm{s} \mathrm{NRZ} \mathrm{data} \mathrm{(PRBS} 2^{31}-1$ ) at a BER of $1 \times 10^{-9}$

ONU data to be recovered, $2 \times$ down-sampling and OFDM signal recovery, including an ultra-low overhead $(0.001 \%)$ pilot-subcarrier based channel estimation/equalization function and a zero overhead symbol alignment algorithm. It should also be noted the ONUs and OLT use a common $2 \mathrm{GHz}$ sample clock providing zero sampling frequency offset.

In performing numerical simulations, an IMDD DFMA PON theoretical model detailed in [3] is adopted, where an approach published in [8] is employed to simulate the OOFDM signal generation, nonlinear transmission and direct detection. Here all experimentally measurable transceiver/PON parameters used in [4] are also adopted, which are summarized in Table I. In numerical simulations, different channel roll-off levels are realized by varying the LPF profiles. For simplicity, the roll-off value, $R(\mathrm{~dB})$, is defined as the LPF attenuation at the Nyquist frequency ( $1 \mathrm{GHz}$ for all the cases considered here). The various LPF magnitude responses used in the performance analysis are plotted in Fig. 2. The optical signal extinction ratio (ER) [9] in each ONU is finely adjusted to ensure good agreement between numerical simulations and experimental measurements for all different scenarios. The introduction of the LPF profiles and suitable signal ERs allows the utilization of an ideal intensity modulator in each ONU [7]. As a direct result, the intensity

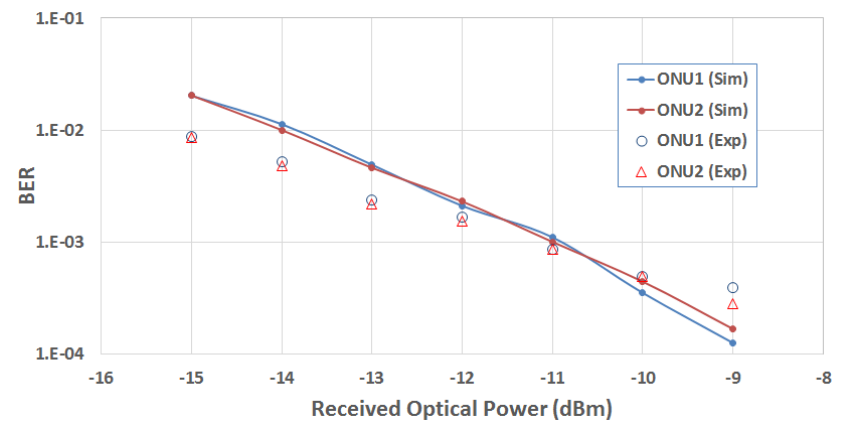

Fig. 3. Comparisons of BER versus received optical power performance between simulations and experiments. (Sim: simulations, Exp: experiment). 
modulation-induced frequency chirp is also negligible.

\section{PARAMETER IdENTIFICATION AND SimUlation Model VALIDATION}

To identify the optical signal ER and subsequently verify the above-described numerical simulation models, BER versus ROP performance comparisons are made between numerical simulations and experimental results [4] by taking into account all the aforementioned transceiver/PON parameters and the experimentally adopted conditions including $R=10 \mathrm{~dB}$ and the six highest frequency subcarriers enabled. The comparisons are shown in Fig.3, in obtaining which the optical signal ER is finely adjusted until the simulated results closely match all the experimental measurements. The resulting optical signal ER is $0.49 \mathrm{~dB}$, which is taken to be a constant value throughout the Letter. It can be seen in Fig. 3 that a good match between simulated and experimental results is obtained across the entire dynamic ROP range. In addition to the BER versus ROP performance, good agreements between numerically simulated results and experimental measurements are also achieved in terms of upstream ONU transmission capacity, ONU LPV range and inter-ONU STO, as detailed in Section IV. The above discussions confirm not only the accuracy of the identified ER parameter but also the validity of the numerical simulation models.

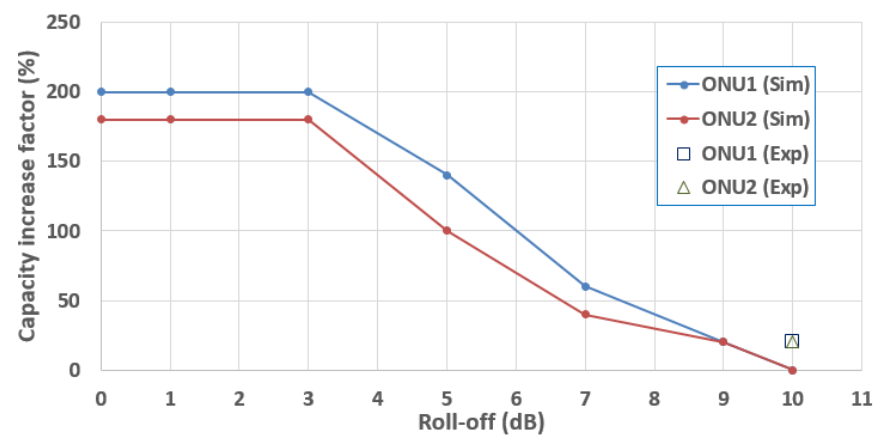

Fig. 4. Capacity increase ratio of each ONU versus channel roll-off

\section{UPSTREAM ONU PERFORMANCE TOLERANCE TO CHANNEL ROLL-OFF}

By making use of the verified numerical models and the identified ER parameter, in this section detailed explorations are undertaken numerically to investigate the upstream IMDD DFMA PON performance tolerance to channel roll-off. Fig. 4 presents the simulated channel roll-off-dependent ONU upstream transmission capacity together with experimentally measured results. For each ONU, the signal transmission capacity is calculated by considering the subcarriers with BERs of $\leq 4 \times 10^{-3}$ (FEC limit) only. Fig. 4 is plotted using a $0.625 \mathrm{~Gb} / \mathrm{s}$ raw bit rate per ONU as reference, where $R=10 \mathrm{~dB}$ and the 5 highest frequency subcarriers are enabled.

It can be seen in Fig. 4 that the ONU transmission capacity can be increased by $200 \%$ (180\%) for ONU1 (ONU2), when $R$ is reduced from $10 \mathrm{~dB}$ to $\leq 3 \mathrm{~dB}$. This is because the DFMA signal generation produces a double sideband spectrum, as shown in the inset of Fig. 2, with the original signal spectrum mapped to both the upper and lower sidebands. The channel roll-off effect introduces amplitude variations between the corresponding frequency components in the upper and lower sidebands, this prevents full cancellation between the sidebands of the unwanted signal when these sidebands are mapped back in the DFMA receiver. As such, the CCI effect occurs. In particular, a larger roll-off causes a greater amplitude variation between the two sidebands, thus lower frequency subcarriers suffer more CCI and more low frequency subcarriers' BERs exceed the FEC limit as the roll-off increases [6]. The ONU transmission capacities saturate to maximum when $R \leq 3 \mathrm{~dB}$, where ONU2 can support at most 14 (of 15) subcarriers as the first subcarrier must be dropped due to the high attenuation of its sine filter near the DC component.

As the impact of channel roll-off also depends upon actual signal bandwidth, performance analysis is performed for the cases of both the 6 and 12 highest frequency subcarriers enabled. Channel bandwidth utilisation factor $\alpha$ is thus defined as the ratio of useful information-carrying signal bandwidth $B_{S I G}$ to maximum available channel bandwidth $B_{C H}$ determined by the Nyquist theorem. For the considered OOFDM cases, $B_{S I G}$ is approximated as $2(N+1) \Delta f$, where $N$ is the number of enabled subcarriers and $\Delta f$ is the subcarrier frequency spacing. As $B_{C H}=1 \mathrm{GHz}$ and $\Delta f$ is $31.25 \mathrm{MHz}$, the value of $\alpha$ is $0.43(0.81)$ when 6 (12) subcarriers are enabled.

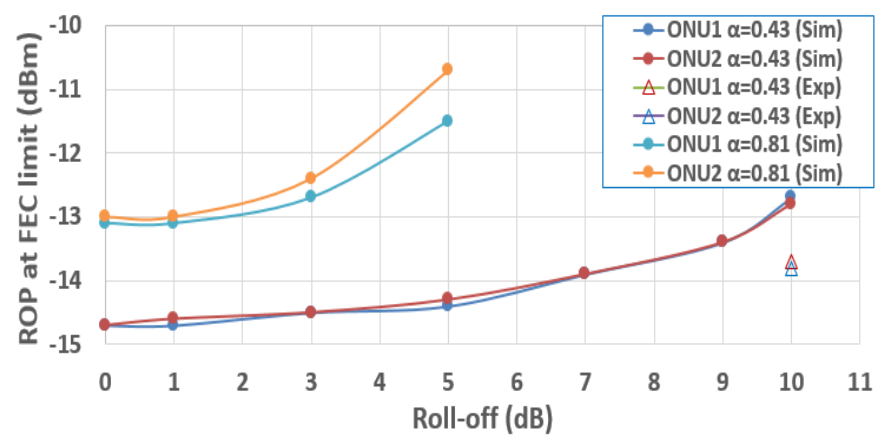

Fig. 5. Received optical power at FEC limit versus roll-off.

Fig. 5 shows the minimum required ROP at the adopted FEC limit versus roll-off for $\alpha=0.43$ and 0.81 . Again, very similar experimental results are also obtained in the same figure. When $\alpha=0.43(0.81)$, there is a $\sim 2 \mathrm{~dB}$ power penalty when $R=10 \mathrm{~dB}$ $(5 \mathrm{~dB})$ relative to $R=0 \mathrm{~dB}$. This indicates that as $\alpha$ increases the ROP sensitivity to roll-off increases. This is due to higher CCIs associated with lower frequency subcarriers, thus the ROP must grow as roll-off and/or $\alpha$ increases in order to maintain the required optical signal-to-noise ratio (OSNR) at the FEC limit.

Fig. 6 shows the ONU LPV range versus channel roll-off. The ONU LPV range is defined as the maximum allowable variation in an ONU's optical launch power for a fixed ROP at the OLT, which maintains the BERs of all ONUs below the adopted FEC limit. In obtaining Fig. 6, ONU1's optical launch power is varied from an initial value of $4 \mathrm{dBm}$, whilst ONU2's is fixed at $4 \mathrm{dBm}$ and the ROP is held at $-9 \mathrm{dBm}$. As ROP is

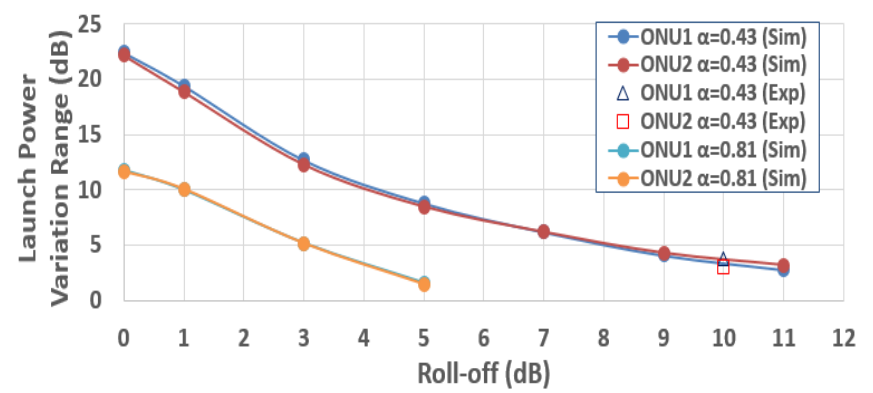

Fig. 6. ONU Launch power variation range versus roll-off. 
fixed the LPV range limits are found to be roughly symmetrical about $4 \mathrm{dBm}$. The experimental results plotted in Fig. 6 show excellent correlation with numerical simulations. Fig.6 shows a fairly rapid reduction in ONU LPV range as roll-off increases. This is due to the fact that as the ROP from ONU1 increases the ROP from ONU2 decreases, thus ONU1's (ONU2's) CCI from ONU2 decreases, (ONU1 increases). This effect is magnified for higher roll-off-induced CCI. Also, there is a $\sim 8 \mathrm{~dB}$ reduction in ONU LPV range for a given roll-off for $\alpha=0.81$ compared to $\alpha=0.43$, thus ONU LPV range is also sensitive to $\alpha$.

As channel orthogonality is dependent on sample timing synchronization between two spectrally-overlapped orthogonal channels, numerical explorations are also made of the upstream performance tolerance to inter-ONU STO (IOS) at various rolloffs. Here the IOS range is defined as the maximum signal time delay adjustment range of one ONU to maintain both ONU BERs below the adopted FEC limit. To finely control the IOS, in each ONU the electrical signal after the DAC is firstly upsampled from $2 \mathrm{GS} / \mathrm{s}$ to $40 \mathrm{GS} / \mathrm{s}$. Then an adjustment of the time delay is made to the electrical signal from ONU2 at a resolution of 25ps. In the OLT, after the ADC, the digital signal is downsampled from $40 \mathrm{GS} / \mathrm{s}$ back to $2 \mathrm{GS} / \mathrm{s}$. To find the required receiver STO, a comprehensive sweep across two consecutive original sample intervals totaling $1 \mathrm{~ns}$ is conducted in order to compare the BER against receiver STO for each ONU. To determine the required receiver STO, two approaches are investigated: i) select the same receiver STO for both ONUs with their BERs kept minimized and as close as possible, referred to as "balanced BER", and ii) select separate receiver STO for each ONU for minimum BERs, referred to as "optimum BER". The first approach is necessary when both channels are received in the same transceiver incorporating a common ADC, and the second approach is applicable when an independent ADC is employed for each channel.

For the abovementioned two approaches, examples of BER versus receiver STO are plotted in Fig.7, and the corresponding IOS range variations with roll-off are shown in Fig. 8, where again the experimental results match very well the simulations.

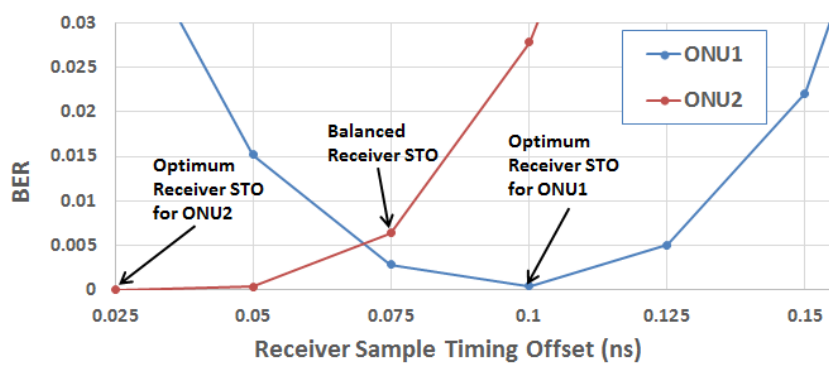

Fig. 7. Example of ONU BER versus receiver STO when the inter-ONU STO is $0.075 \mathrm{~ns}$ at $10 \mathrm{~dB}$ roll-off and $\alpha=0.43$.

The results in Fig. 8 reveal that: i) a sharp roll-off-induced large CCI leads to a reduced IOS range; ii) the IOS range is significantly lower for the balanced case, thus employing independent channel ADCs considerably reduces timing synchronization requirements between ONUs, and iii) a high $\alpha$ value reduces the IOS range for a specific roll-off, thus the maximum required $\alpha$ value dictates the maximum roll-off for a fixed IOS range. The worst-case IOS range in Fig. $8(0.1 \mathrm{~ns})$ is easily accommodated with modern timing delay circuits, however a larger IOS range is still preferred as it relaxes the requirements on tracking speed and complexity of the ONU timing control algorithms.

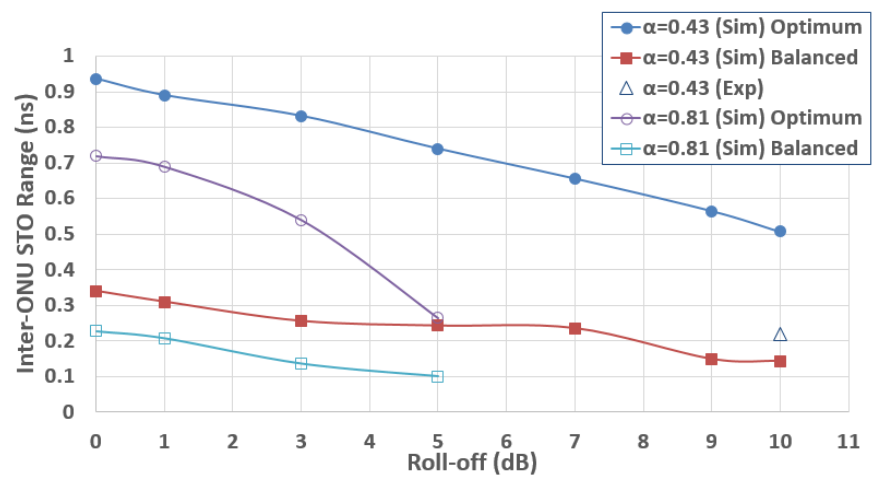

Fig. 8. Inter-ONU STO range versus roll-off.

\section{CONCLUSIONS}

The channel roll-off effect is a key system feature impacting IMDD DFMA PON performance and as channel roll-off severity is highly design dependent, it is a key issue for component, transceiver and system designers to understand when balancing cost and performance. The tolerance of various performance characteristics to varying degrees of channel rolloff have therefore been fully investigated in a numerically simulated upstream IMDD DFMA PON, validated by strong agreement with experimentally obtained results. These results therefore provide important insights to facilitate the effective design of DFMA transceivers and PONs.

\section{REFERENCES}

[1] G. Talli, et al., "SDN enabled dynamically reconfigurable high capacity optical access architecture for converged services," J. Lightw. Technol., vol. 35, no. 3, pp. 550-560, Feb. 1, 2017.

[2] K. Kondepu, et al., "Exploiting SDN for integrating green TWDM-PONS and metro networks preserving end-to-end delay," IEEE/OSA J. Opt. Commun. Netw., vol. 9, no. 1, pp. 67-74, Jan. 2017.

[3] M. Bolea, R. P. Giddings, M. Bouich, C. Aupetit-Berthelemot, and J.M. Tang, "Digital filter multiple access PONs with DSP-enabled software reconfigurability," IEEE/OSA J. Opt. Commun. Netw., vol. 7, no. 4, pp. 215-222, Apr. 2015.

[4] X. Duan, R.P. Giddings, S. Mansoor and J.M. Tang, "Experimental demonstration of upstream transmission in digital filter multiple access PONs with real-time reconfigurable optical network units," IEEE/OSA J. Opt. Commun. Netw., vol. 9, no. 1, pp. 45-52, Jan. 2017.

[5] W. Jin, X. Duan, Y. Dong, B. Cao, R. P. Giddings, C. F. Zhang, K. Qiu, and J. M. Tang, "DSP-enabled flexible ROADMs without optical filters and O-E-O conversions," J. Lightw. Technol. vol. 33, no. 19, pp. 41244131, 2015.

[6] E. Al-Rawachy, R. P. Giddings, J. M. Tang, "Experimental demonstration of a DSP-based cross-channel interference cancellation technique for application in digital filter multiple access PONs," Optics Express, vol. 25, no. 4, pp. 2850-3862, Feb, 2017.

[7] Y. Dong, E. Al-Rawachy, R.P. Giddings, W. Jin, D. Nesset, J.M. Tang, "Multiple channel interference cancellation of digital filter multiple access PONs," J. Lightw. Technol., vol. 35, no. 1, pp. 34-44, Jan. 1, 2017.

[8] J. M. Tang and K. A. Shore, "30-Gb/s signal transmission over $40-\mathrm{km}$ directly modulated DFB-laser-based single-mode-fiber links without optical amplification and dispersion compensation," J. Lightw. Technol., vol. 24, no. 6, pp. 2318-2327, June 2006.

[9] J. L. Wei, A. Hamie, R. P. Giddings and J. M. Tang, "Semiconductor optical amplifier-enabled intensity modulation of adaptively modulated optical OFDM signals in SMF-based IMDD systems," J. Lightw. Technol., vol. 27, no. 16, pp. 3678-3688, Aug. 15, 2009. 